\title{
Histone Methyltransferase SETD2 in Lymphoid Malignancy
}

\author{
Weijie Li \\ Department of Pathology and Laboratory Medicine, Children's Mercy Hospital, \\ University of Missouri-Kansas City School of Medicine, Kansas City, USA
}

Author for correspondence: Weijie Li, Department of Pathology and Laboratory Medicine, Children's Mercy Hospital, Kansas City, USA. Email: wli@cmh.edu

Cite this chapter as: Li W. Histone Methyltransferase SETD2 in Lymphoid Malignancy. In: Gallamini A, Juwied M. editors. Lymphoma. Brisbane (AU): Exon Publications. Online first 2021 Nov 21.

Doi: https://doi.org/10.36255/exon-publications.lymphoma.2021.

setd2-lymphoid-malignancy

\begin{abstract}
SETD2 (SET Domain Containing 2, Histone Lysine Methyltransferase) is the only human gene encoding the histone methyltransferase responsible for trimethylation of lysine 36 of histone H3. SETD2 protein is involved in multiple important cellular processes that include transcriptional regulation, DNA damage repair, alternative RNA splicing, genomic stability, apoptotic response, and interferon response. As a tumor suppressor, SETD2 loses its activities by loss-offunction mutations in a wide spectrum of tumors. The alterations of SETD2 are the most common genetic changes detected in monomorphic epitheliotropic intestinal T-cell lymphoma, seen in over 90\% of the cases. SETD2 is the commonest silenced gene detected in hepatosplenic T-cell lymphoma, seen in $25 \%$ of the cases. SETD2 alterations have been detected in approximately 10\% of precursor B-cell lymphoblastic leukemia/lymphoma cases, and commonly gained in the relapse of this disease. SETD2 alterations have also been found in about $10 \%$ of early-T-precursor lymphoblastic leukemia, up to $10 \%$ of diffuse large B-cell lymphoma, up to $7 \%$ of chronic lymphoblastic leukemia/small lymphocytic lymphoma, and in a small portion of other lymphoid malignancies. Experimental loss
\end{abstract}

In: Gallamini A, Juwied M (Editors). Lymphoma. Exon Publications, Brisbane, Australia. ISBN: 978-0-6453320-0-1; Doi: https://doi.org/10.36255/exon-publications.lymphoma.2021

Copyright: The Authors.

License: This open access article is licenced under Creative Commons Attribution-NonCommercial 4.0 International (CC BY-NC 4.0) https://creativecommons.org/licenses/by-nc/4.0/ 
of SETD2 can promote tumor cell proliferation and result in chemotherapy resistance. Targeting SETD2 may offer a potential therapeutic strategy for these lymphoid malignancies.

Keywords: hepatosplenic T-cell lymphoma; histone methyltransferase; lymphoblastic leukemia; monomorphic epitheliotropic intestinal T-cell lymphoma; SETD2

\section{INTRODUCTION}

Chromatin formation is a major mechanism by which eukaryotic cells regulate gene transcription. Nucleosome is the basic unit of chromatin. It consists of $\sim 147$ base pairs of DNA wound around eight histone proteins: $2 \mathrm{H} 2 \mathrm{~A}, 2 \mathrm{H} 2 \mathrm{~B}, 2 \mathrm{H} 3$, and $2 \mathrm{H} 4$ (1). Post-translational histone modifications play critical roles in the regulation of human genome (2). Histone lysine methylation can be associated with either active or repressed state of gene transcription, depending on the methylation status of the lysine residue (mono-, di-, or tri-) (3). In general, lysine methylation functions to recruit effector proteins with domains capable of recognizing these modified sites and adjusting nucleosome-DNA interactions (4). Histone H3 lysine 36 methylation (H3K36me) is one of the methylated lysine residues well studied and conserved from yeast to human. Although many histone methyltransferases (KMTs) can catalyze H3K36 to generate monomethylated histone H3 (H3K36mel) or dimethylated histone H3 (H3K36me2), SETD2 (SET Domain Containing 2, Histone Lysine Methyltransferase) is the only mammalian HMT that catalyzes H3K36 trimethylation (H3K36me3).

SETD2 was first isolated from human hematopoietic stem cells, and it was thought to be associated with Huntington's disease (5). SETD2 protein is involved in multiple important cellular processes, such as transcriptional regulation, DNA damage repair, alternative RNA splicing, genomic stability, and apoptotic response (Figure 1). Mutations of SETD2 have been detected in various tumors $(6,7)$. This chapter introduces the structure and functions of SETD2, and reviews the genetic alterations and possible pathogenetic roles of SETD2 in lymphoid malignancy.

\section{PROTEIN STRUCTURE AND FUNCTIONS OF SETD2}

Many studies have been dedicated to SETD2 since its discovery twenty years ago. Currently, the gene and protein structures of SETD2 are well defined, and the biological functions of SETD2 are well studied.

\section{Protein structure}

The human SETD2 gene is located on p21.31 of chromosome 3, a region frequently targeted by copy number loss in a wide spectrum of tumors (8). SETD2 gene occupies a genomic region of $147 \mathrm{~Kb}$ and is composed of 26 exons encoding three mRNA transcript variants ranging from 8,452 to 8,737 nucleotides in length. 


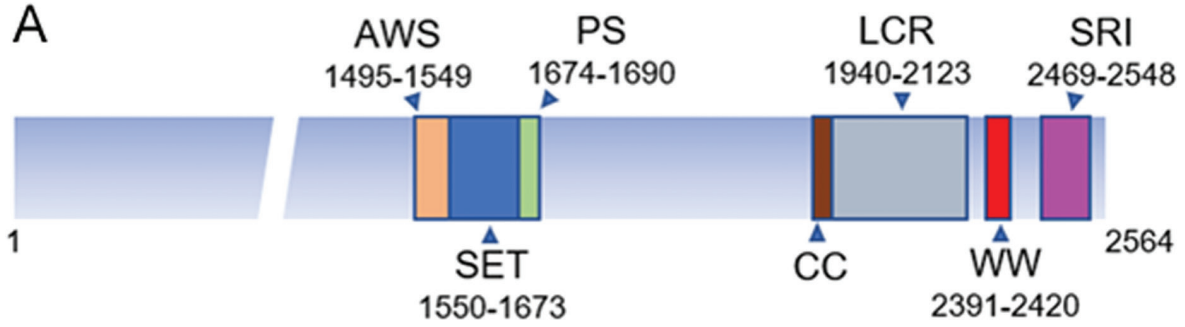

B

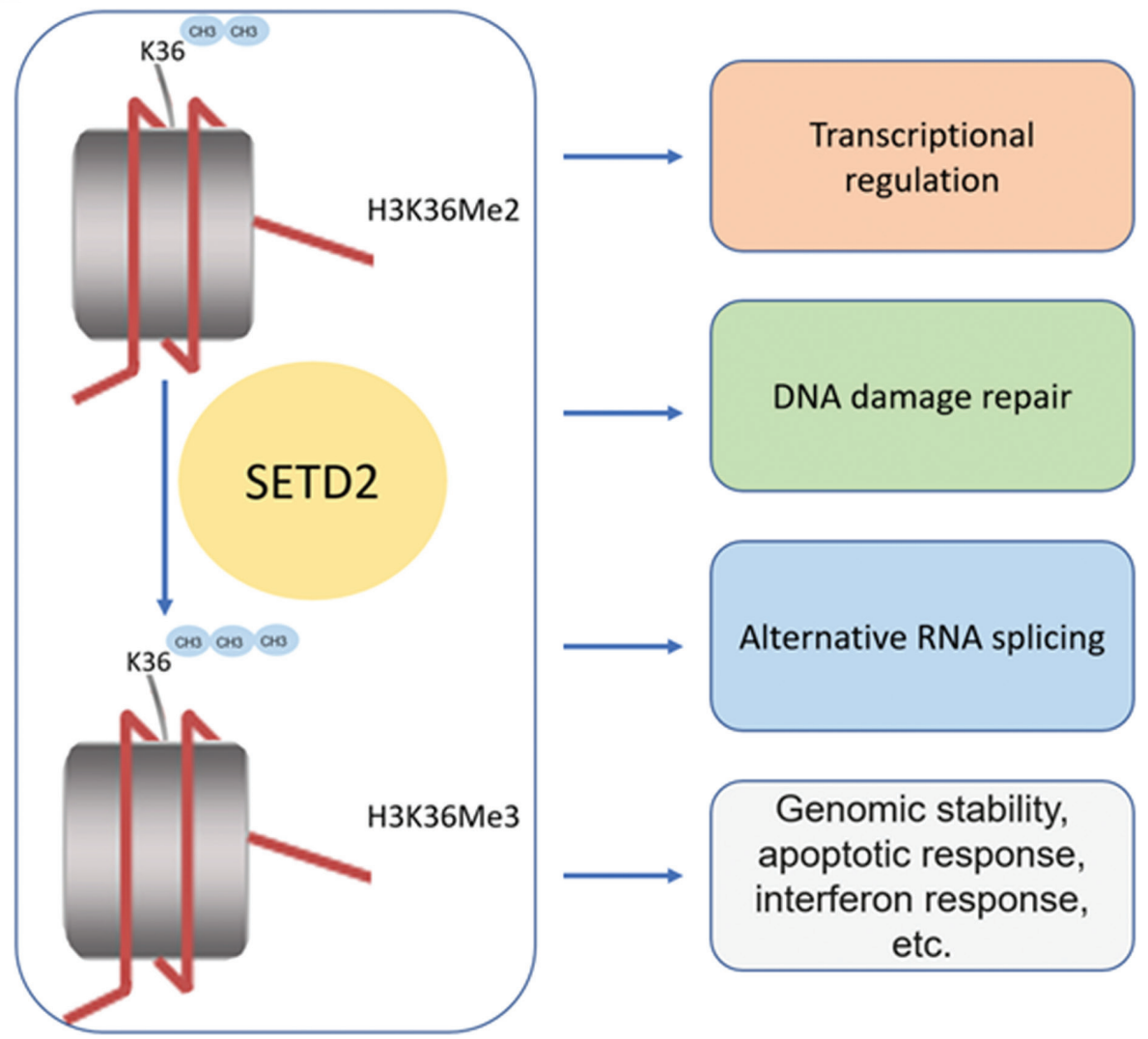

Figure 1. The structure and cellular functions of SETD2 protein. A, SETD2 protein is composed of 2,564 amino acids and contains three main conserved functional domains: AWS-SET-PS, WW and CC domains, and SRI. B, SETD2 protein is a histone methyltransferase that specifically trimethylates 'Lys-36' of histone H3 (H3K36me3) using dimethylated 'Lys-36' (H3K36me2) as substrate. It has multiple cellular functions that include transcriptional regulation, DNA damage repair, alternative RNA splicing, keeping genomic stability, regulating apoptotic response, involving in interferon response, etc. AWS, associated with SET; CC, Coiled-Coiled; PS, post-SET; SETD2, SET domain-containing 2; SRI, Set2-Rpb1 interacting. 
Human SETD2 protein has two main isoforms. Isoform 1 is composed of 2,564 amino acids with a molecular weight of $287.5 \mathrm{kDa}$. Isoform 2 is 44 residues shorter than isoform 1 at the N-terminus and is composed of 2520 amino acids with a molecular weight of $282.6 \mathrm{kDa}$.

Three conserved functional domains (Figure 1A) have been identified in the SETD2 protein: the AWS (associated with SET)-SET-PS (post SET) domains, WW and CC (coiled-coiled) domains, and SRI (Set2 Rpbl Interacting) domain $(6,7)$. The AWS-SET-PS domain is the catalytic domain performing the H3K36 trimethylation. In yeast, the SET domain is located at the N-terminus of the protein, while human SETD2 has an extended and low complexity N-terminus with no conserved domains and no unknown function (9). The WW domain, located in the C-terminal region of SETD2, contains two conserved tryptophan (W) residues. The WW domain preferentially binds proline-rich segments of other proteins, mediating protein-protein interactions (10). The CC domain is also a conserved protein-protein interaction motif that promotes homo-dimerization of certain proteins. The SRI domain spans a 79-amino-acid region at the C-terminal end of SETD2 and is the primary docking site of RNA polymerase II (RNAPII) (11). SRI domain binds to serine 2 and serine 5 doubly phosphorylated C-terminal domain (CTD) repeats of RNAPII (12).

\section{Transcriptional regulation}

Transcription is a highly regulated process of making a RNA copy of a gene, which directs the synthesis of the encoded protein. Both SETD2 and H3K36me3 are involved in transcription regulation (11-14). The SRI domain of SETD2 protein interacts with the CTD of RNAPII, and plays an important role in regulating the initiation, elongation, and termination of the transcription. SETD2 also plays a role in nucleosome reorganization in bodies of transcribed genes after the passage of RNAPII machinery. Loss of SETD2 can lead to spurious intragenic initiation of transcription through abnormal targeting of the FACT (FAcilitates Chromatin Transcription) complex to chromatin (15). In yeast, H3K36me3 prevents the initiation of spurious intrageneric transcription by recruiting histone deacetylase complexes $(13,16)$. H3K36me3 also recruits DNMT3B (DNA-methyltransferase 3B), which leads to the dense methylation of gene bodies, and thus protects RNAPII from spurious re-initiation of transcription and reinforces intragenic silencing (17).

\section{DNA damage repair}

SETD2 plays an important role in DNA damage repair. DNA damage can be triggered by various causes. Failure of DNA repair can result in cell death, and misrepair can lead to tumorigenesis. DNA double-strand break (DSB) is one of the most significant forms of DNA damages. SETD2 activity is required for DSB repair by recruiting RAD51 and LEDGF (Lens epithelium-derived growth factor) to DSBs and promoting repair of active genes by homologous recombination (18). DNA mismatch repair (MMR) is an important biological pathway that plays a key role in maintaining genomic stability. SETD2 acts as a key regulator of DNA MMR by generating H3K36me3, a mark required to recruit MSH6 subunit of the MutS 
alpha complex. The recruitment of the MutS alpha complex to chromatin to be replicated allows a quick identification of the mismatch DNA to initiate MMR reaction (19). MSH6, like many other H3K36me3 effector proteins, contains a PWWP domain that is essential for H3K36me3 binding. Cells deficient for SETD2 display microsatellite instability and increased mutation frequency.

\section{Alternative splicing}

Post-translational modification of histone tails is associated with the regulation of alternative splicing. Zinc finger MYND-domain containing 11 (ZMYND11) selectively recognizes $\mathrm{H} 3 \mathrm{~K} 36 \mathrm{me} 3$ and regulates intron retention (IR), the least understood RNA alternative splicing event in mammalian cells (20). The mutations of SETD2 are associated with chromatin accessibility changes and RNA processing defects in tumors lacking H3K36me3 (21). PWWP-domain containing proteins play a pivotal role in the regulation of this process. For example, ZMYND11, BRPF1 (Bromodomain and PHD finger-containing 1) and MRG15 (MORF-related gene 15) bind to H3K36me3 and then either interact with components of the RNA splicing machinery or influence transcriptional regulation $(22,23)$. The LEDGF-H3K36me3 interaction can modulate RNA splicing through LEDGFdependent recruitment of spliceosome factors, such as SRSF1 (Serine/argininerich splicing factor 1) (24). Study in yeast (25) showed that mutants lacking H3K36me exhibited reduced splicing efficiency across the genome, and correct splicing was dependent on the association of Set2 with the CTD of RNAPII.

\section{Other functions}

SETD2 also has non-histone-related functions, such as methylating tubulin and maintaining the genome stability by promoting proper chromosome segregation $(26,27)$. Loss of SETD2 leads to genomic instability, cytokinesis defects and mitotic abnormalities $(26,27)$. SETD2 also methylates STAT1 and is important for interferon-mediated antiviral activity (28) and potentially immune surveillance response. In addition, SETD2 can interact with p53 through the WW and SET domains (29). p53 is an important tumor suppressor which regulates cell cycle and apoptosis. SETD2 down-regulates the expression of HDM2 (human double minute 2), which is a p53 negative regulator, and leads to enhanced p53 protein stability (29).

\section{THE ALTERATIONS AND POSSIBLE ROLES OF SETD2 IN LYMPHOID MALIGNANCY}

With the application of next generation sequencing techniques in clinical research, the mutational landscapes of many tumors have been well studied recently. Mutations of SETD2 have been detected in various types of cancers including some lymphomas and leukemia. Most of these mutations result in a loss of SETD2 function, and experimental loss of SETD2 in lymphoma/leukemia cell lines or animal models have demonstrated an increase of tumor cell proliferation, and resistance to certain chemotherapy. 


\section{Monomorphic epitheliotropic intestinal T-cell lymphoma}

Monomorphic epitheliotropic intestinal T-cell lymphoma (MEITL) is a rare peripheral T-cell lymphoma of intestine and was called type II enteropathyassociated T cell lymphoma (EATL) in the previous WHO classification. It is derived from intraepithelial lymphocytes and characterized by an aggressive clinical course. The genetic basis of MEITL is poorly characterized (30). Recent studies (31-33) reveal that mutations of SETD2 predicting a loss of function are the hallmark genetic changes of this disease. The study conducted by the European group (31) identified SETD2 mutations in 19/23 (83\%) MEITL cases, loss of one SETD2 locus in 7/23 (30\%) cases, and overall SETD2 genomic alterations in 21/23 (91\%) cases by whole exon sequencing (WES) or targeted deep sequencing. Eighteen of the total 26 mutations (69\%) identified in this study were nonsense, frameshift indels or splicing mutations predicted to impair protein function and distributed along the whole gene. This study also showed that SETD2 mutations correlated with complete loss or severe reduction in $\mathrm{H} 3 \mathrm{~K} 36 \mathrm{me} 3$, suggesting that these mutations were indeed loss of function. Other common genetic alterations identified in this study were mutations related to JAK-STAT pathway, which are also commonly seen in EATL. SETD2 alterations were not seen in any of the 8 EATL cases included in this study. A Japanese group has conducted a similar study on 9 Japanese MEITL cases and found similar results; all the cases showed alterations of SETD2 (mutations and/or loss of the corresponding 3p21 locus), and all the SETD2mutated cases demonstrated loss of H3K36me3 by IHC staining (32).

Moffitt et al (33) studied the genetic changes of 23 MEITL cases and 41 EATL cases by WES and identified SETD2 mutations in 70\% of MEITL cases and less than $15 \%$ of EATL cases. Most of the mutations were nonsense, frameshift insertions or deletions, and some cases had multiple mutations in SETD2, supporting its role as a tumor suppressor gene in these lymphomas.

\section{Hepatosplenic T-cell lymphoma}

Hepatosplenic T cell lymphoma (HSTL) is a rare subtype of extranodal lymphoma that predominantly involves liver and spleen without lymphadenopathy. The lymphoma cells are phenotypically cytotoxic T cells and commonly of gamma delta T-cell origin (30). The genetic drivers of this disease are unknown. McKinney et al (34) studied 68 HSTL cases by WES and discovered that SETD2 was the most frequently silenced gene. SETD2 was mutated in $25 \%$ of all the cases and most ( $71 \%)$ of the cases contained at least one loss of function (nonsense or frameshift) mutations. The mutations occurred predominantly in exons 3, 10-12 and 19-21, with the SRI domain as the most frequently mutated domain. There was no significant survival difference identified between SETD2 altered and unaltered patients. Knockdown of SETD2 by shRNAs targeting SETD2 in HSTL cell lines promoted cell growth and altered the expression of cell-cycle genes. These results indicate that SETD2 is a tumor suppressor gene in HSTL.

Given that SETD2 alterations are a hallmark genetic finding of MEITL, another rare peripheral T-cell lymphoma commonly originated from gamma delta T-cells, it is speculated that SETD2 gene may play a role in T-cell development. The study of Moffitt et al (33) showed that high level expression of SETD2 in the immature thymocytes of CD4/8 double-positive CD69-negative stage is a critical step in confirming the $\alpha \beta$ T cell lineage choice. The conditional SETD2 knockout mouse model in this study 
showed that SETD2 loss in T cells was associated with an expansion of $\gamma \delta$ T cells, which confirms that SETD2 plays an important role in controlling the destiny of a thymocyte precursor to become an $\alpha \beta$ T cell. The lack of lymphoma development in SETD2 knockout mice in this study suggests that the development of HSTL or MEITL requires the activation of other collaborating genetic events. More work is needed to fully define the role of SETD2 in the oncogenesis of these lymphomas.

\section{Lymphoblastic leukemia/lymphoma}

B-lymphoblastic leukemia/lymphoma (B-ALL/LBL) is the most common pediatric lymphoid malignancy, which has a very good prognosis (over 90\% curable rate) with current risk-adjusted treatment regimen. However, $15-20 \%$ of the cases will relapse and the clinical outcome of the relapsed cases is poor. Mar et al (35) identified SETD2 mutations in $12 \%$ of de novo pediatric B-ALL/LBL patients by PCR and Illumina sequencing techniques. SETD2 mutations were more prevalent in the KMT2A rearranged (22\%) and ETV6-RUNX1 rearranged (13\%) subtypes. These leukemia cases also frequently harbored mutations in the Ras signaling pathway. The SETD2 mutations identified were commonly loss of function frameshift or nonsense mutations with no obvious hotspot. Some patients harbored multiple SETD2 mutations. Sequencing of the 30 matched relapsed samples showed a significant gain of mutations in epigenetic regulators including SETD2. Similar finding was also seen in adult B-ALL/LBL (36).

The selective enrichment of alterations in epigenetic regulators in relapse indicates that mutations in genes encoding epigenetic regulators such as SETD2 may be associated with resistance to chemotherapy and/or grow advantage. This hypothesis was recently confirmed in the study of leukemia cell lines and murine leukemia model (37). SETD2 mutations led to resistance to DNA-damaging agents, such as cytarabine, 6-thioguanine, doxorubicin, and etoposide, but not to a non-DNA damaging agent, L-asparaginase. This chemotherapy resistance is most likely due to disorganization of DNA damage response normally mediated by H3K36me3. Heterozygous conditional inactivation of SETD2 in a murine model decreased the latency of KMT2A-AF9-induced leukemia and caused resistance to cytarabine treatment. Interestingly, disruption of both SETD2 alleles (homozygous loss) inhibited leukemogenesis, which was likely because of excessive DNA damage and impaired replication (37). The tumor suppressor role of SETD2 in acute leukemia was also confirmed by another study (38), which revealed that knockdown of SETD2 led to proliferative advantage and accelerated leukemia development of KMT2A-rearranged leukemia cells in vitro and in vivo.

T-ALL/LBL is a neoplasm of lymphoblasts committed to the T-cell lineage, typically composed of medium-sized blasts with scant cytoplasm, dispersed chromatin, inconspicuous or prominent nucleoli (Figure 2A). T-ALL/LBL usually has a worse clinical outcome in children compared to pediatric B-ALL/LBL. Early-T-cell precursor (ETP) ALL is a subtype of T-ALL with a unique immunophenotype indicating only limited early T-cell differentiation. Gene expression profile of ETPALL is resemblant to that of normal early thymocyte precursors and different from that of T-ALL/LBL corresponding to later maturational stages. The mutational profile of ETP-ALL is close to that of myeloid leukemias (30). SETD2 mutations were identified in $10 \%$ of ETP-ALL cases but were rare in non-ETP T-ALL/LBL cases (39). In our very limited number of T-ALL/LBL cases with next generation sequencing data, a pathogenic loss-of-function mutation-c.4715 C>A 
A

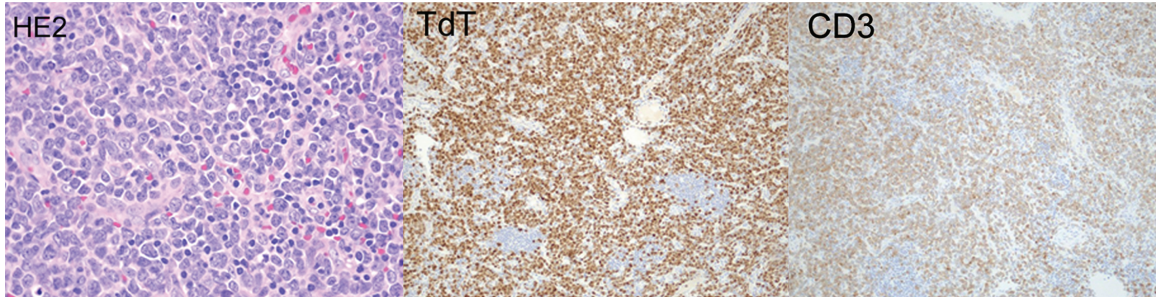

B
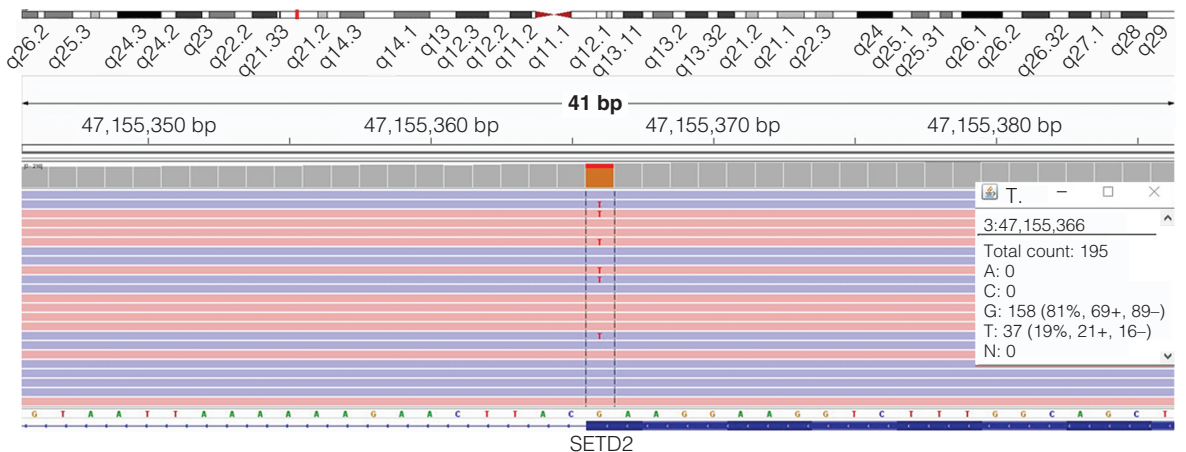

Figure 2. Histopathology and SETD2 mutation of a T lymphoblastic lymphoma case.

A, Hematoxylin and eosin-stained section shows sheets of medium to large-sized atypical lymphoid cells with round, oval or irregular nuclei, fine nuclear chromatin, distinct one or multiple nucleoli and scant cytoplasm. Mitosis is easily seen. The tumor cells are positive for CD3 and TdT. B, Next generation sequencing (whole exome sequencing with an average depth of coverage of 489x) shows a pathogenic loss-of-function mutation: c.4715 C>A (p.Ser1572*) at a VAF of $19 \%$ in SETD2.

(p.Ser1572*) (Figure 2B) at a VAF of 19\% in SETD2-was found in a T-LBL case, which showed near-ETP ALL phenotype and interfollicular growth pattern (40).

\section{Mature B-cell lymphoma}

Chronic lymphocytic leukemia/small lymphocytic lymphoma (CLL/SLL) is a neoplasm composed of monomorphic small mature B cells coexpressing CD5 and CD23. CLL is the most common leukemia in Western countries. Using high-resolution single nucleotide polymorphism (SNP) arrays, Parker et al (41) identified recurrent deletions of the SETD2 locus in 8/261 (3\%) CLL patients. The SETD2 deletions were associated with loss of TP53, genomic complexity and chromothripsis. Using next-generation sequencing, they also detected mutations of SETD2 in an additional 3.8\% of patients (23/602). SETD2 deletions or mutations were found as a mono-allelic lesion and were associated with reduced mRNA expression of SETD2. They also found that patients with SETD2 abnormalities and wild-type TP53 and ATM had reduced progression-free and overall survival compared with cases wild type for all three genes. The frequency of SETD2 abnormalities in CLL/SLL seems lower in other studies (Figure 3A). 
A

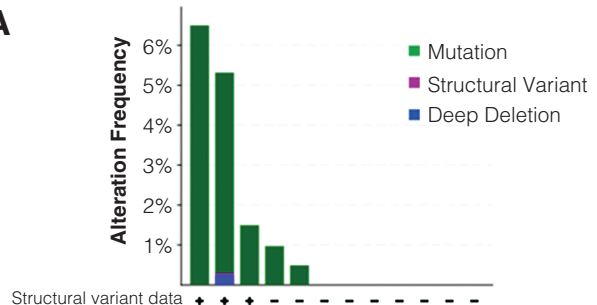

Mutation data ++++++++++++

CNA data $-+-\div-\div+\div++4+$

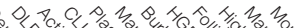

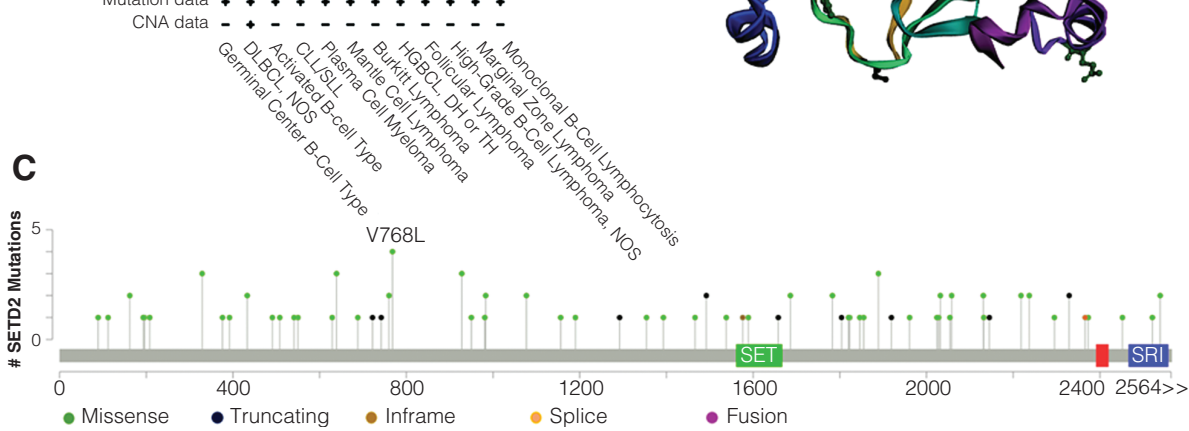

Figure 3. Genetic alterations of SETD2 in mature B-cell lymphomas. Data were retrieved from cBioPortal (http://www.cbioportal.org) in October 2021, including 3338 patients/samples from 10 studies. A. Frequency of SETD2 gene alterations in various types of mature B-cell lymphomas. B, Cartoon crystal structure of SETD2 bound to histone h3.3 k36i peptide. The protein is colored with a rainbow gradient from red (N-terminus) to blue (C-terminus). Mutation types and corresponding color codes are as follows: dark green - missense mutations; black - truncating mutations; brown - inframe mutations. C, Schematic representation of SETD2 mutations associated with mature B-cell lymphomas. Mutation diagram circles are colored with respect to the corresponding mutation types. In case of different mutation types at a single position, color of the circle is determined with respect to the most frequent mutation type. CLL/SLL, chronic lymphocytic leukemia/small lymphocytic lymphoma; DLBCL, diffuse large B-cell lymphoma; SET, Su(var)3-9, enhancer-of-zeste and trithorax; SRI, Set2 Rpb1 interacting.

Diffuse large B-cell lymphoma (DLBCL) is the most common B-cell lymphoma in adults. This disease exhibits prominent heterogeneity in biology and clinical outcome. WES study (42) had identified SETD2 mutations in up to 10\% of DLBCL cases. SETD2 mutations are more common in germinal center B-cell type than those in activated B-cell type (Figure 3A). The majority of the mutations were missense or truncating and distributed along the whole gene (Figure 3B and 3C). SETD2 alterations were also detected in a small portion of plasma cell myeloma cases, but were rare or not detected in Burkitt lymphoma, follicular lymphoma, mantle cell lymphoma, marginal zone lymphoma or monoclonal B-cell lymphocytosis (Figure 3A).

\section{CONCLUSION}

SETD2 is a non-redundant H3K36-specific trimethyltransferase involving in multiple important cellular processes. Mutations and deletions of SETD2 have been 
detected in a number of lymphoid malignancies. Recent studies have suggested or proved the tumor suppressor role of SETD2 in these lymphoid malignancies, especially in MEITL, HSTL and B-ALL/LBL. The SETD2 mutations are enriched in relapse and are associated with chemotherapy resistance in some lymphoblastic leukemia cases. Specific treatment targeting SETD2 may provide a potential therapeutic strategy for these lymphoid malignancies in the future.

Conflict of Interest: The author declares no potential conflicts of interest with respect to research, authorship and/or publication of this manuscript.

Copyright and Permission Statement: The authors confirm that the materials included in this chapter do not violate copyright laws. Where relevant, appropriate permissions have been obtained from the original copyright holder(s), and all original sources have been appropriately acknowledged or referenced.

\section{REFERENCES}

1. Kornberg RD, Lorch Y. Twenty-five years of the nucleosome, fundamental particle of the eukaryote chromosome. Cell. 1999;98(3):285-94. https://doi.org/10.1016/S0092-8674(00)81958-3

2. Bannister AJ, Kouzarides T. Regulation of chromatin by histone modifications. Cell research. 2011; 21(3):381-95. https://doi.org/10.1038/cr.2011.22

3. Black JC, Van Rechem C, Whetstine JR. Histone lysine methylation dynamics: establishment, regulation, and biological impact. Molecular cell. 2012;48(4):491-507. https://doi.org/10.1016/j. molcel.2012.11.006

4. Tessarz P, Kouzarides T. Histone core modifications regulating nucleosome structure and dynamics. Nature reviews Molecular cell biology. 2014;15(11):703-08. https://doi.org/10.1038/nrm3890

5. Faber PW, Barnes GT, Srinidhi J, Chen J, Gusella JF, MacDonald ME. Huntingtin interacts with a family of WW domain proteins. Hum Mol Genet. 1998;7(9):1463-74. https://doi.org/10.1093/ $\mathrm{hmg} / 7.9 .1463$

6. McDaniel SL, Strahl BD. Shaping the cellular landscape with Set2/SETD2 methylation. Cell Mol Life Sci. 2017;74(18):3317-334. https://doi.org/10.1007/s00018-017-2517-x

7. Li J, Duns G, Westers H, Sijmons R, van den Berg A, Kok K. SETD2: an epigenetic modifier with tumor suppressor functionality. Oncotarget. 2016;7(31):50719-34. https://doi.org/10.18632/ oncotarget.9368

8. Kok K, Naylor SL, Buys CH. Deletions of the short arm of chromosome 3 in solid tumors and the search for suppressor genes. Adv Cancer Res. 1997;71:27-92. https://doi.org/10.1016/ S0065-230X(08)60096-2

9. Hacker KE, Fahey CC, Shinsky SA, Chiang YJ, DiFiore JV, Jha DK, et al. Structure/Function Analysis of Recurrent Mutations in SETD2 Protein Reveals a Critical and Conserved Role for a SET Domain Residue in Maintaining Protein Stability and Histone H3 Lys-36 Trimethylation. J Biol Chem. 2016;291(40):21283-95. https://doi.org/10.1074/jbc.M116.739375

10. Gao YG, Yang H, Zhao J, Jiang YJ, Hu HY. Autoinhibitory structure of the WW domain of HYPB/SETD2 regulates its interaction with the proline-rich region of huntingtin. Structure. 2014;22(3):378-86. https://doi.org/10.1016/j.str.2013.12.005

11. Li J, Moazed D, Gygi SP. Association of the histone methyltransferase Set2 with RNA polymerase II plays a role in transcription elongation. J Biol Chem. 2002;277(51):49383-8. https://doi.org/10.1074/ jbc.M209294200

12. Li M, Phatnani HP, Guan Z, Sage H, Greenleaf AL, Zhou P. Solution structure of the Set2-Rpbl interacting domain of human Set2 and its interaction with the hyperphosphorylated C-terminal domain of Rpbl. Proc Natl Acad Sci U S A. 2005;102(49):17636-41. https://doi.org/10.1073/pnas.0506350102 
13. Kizer KO, Phatnani HP, Shibata Y, Hall H, Greenleaf AL, Strahl BD. A novel domain in Set2 mediates RNA polymerase II interaction and couples histone H3 K36 methylation with transcript elongation. Mol Cell Biol. 2005;25(8):3305-16. https://doi.org/10.1128/MCB.25.8.3305-3316.2005

14. Fuchs SM, Kizer KO, Braberg H, Krogan NJ, Strahl BD. RNA polymerase II carboxyl-terminal domain phosphorylation regulates protein stability of the Set2 methyltransferase and histone $\mathrm{H} 3$ di- and trimethylation at lysine 36. J Biol Chem. 2012;287(5):3249-56. https://doi.org/10.1074/jbc. M111.273953

15. Carvalho S, Raposo AC, Martins FB, Grosso AR, Sridhara SC, Rino J, et al. Histone methyltransferase SETD2 coordinates FACT recruitment with nucleosome dynamics during transcription. Nucleic Acids Res. 2013;41(5):2881-93. https://doi.org/10.1093/nar/gks1472

16. Carrozza MJ, Li B, Florens L, Suganuma T, Swanson SK, Lee KK, et al. Histone H3 methylation by Set2 directs deacetylation of coding regions by Rpd3S to suppress spurious intragenic transcription. Cell. 2005;123(4):581-92. https://doi.org/10.1016/j.cell.2005.10.023

17. Neri F, Rapelli S, Krepelova A, Incarnato D, Parlato C, Basile G, et al. Intragenic DNA methylation prevents spurious transcription initiation. Nature. 2017;543(7643):72-77. https://doi.org/10.1038/ nature 21373

18. Carvalho S, Vítor AC, Sridhara SC, Martins FB, Raposo AC, Desterro JM, et al. SETD2 is required for DNA double-strand break repair and activation of the p53-mediated checkpoint. Elife. 2014;3:e02482. https://doi.org/10.7554/eLife.02482

19. Li F, Mao G, Tong D, Huang J, Gu L, Yang W, et al. The histone mark H3K36me3 regulates human DNA mismatch repair through its interaction with MutSo. Cell. 2013;153(3):590-600. https://doi. org/10.1016/j.cell.2013.03.025

20. Guo R, Zheng L, Park JW, Lv R, Chen H, Jiao F, et al. BS69/ZMYNDll reads and connects histone H3.3 lysine 36 trimethylation-decorated chromatin to regulated pre-mRNA processing. Molecular cell. 2014;56(2):298-310. https://doi.org/10.1016/j.molcel.2014.08.022

21. Simon JM, Hacker KE, Singh D, Brannon AR, Parker JS, Weiser M, et al. Variation in chromatin accessibility in human kidney cancer links H3K36 methyltransferase loss with widespread RNA processing defects. Genome research. 2014;24(2):241-50. https://doi.org/10.1101/gr.158253.113

22. Wen H, Li Y, Xi Y, Jiang S, Stratton S, Peng D, et al. ZMYNDll links histone H3.3K36me3 to transcription elongation and tumour suppression. Nature. 2014;508(7495):263-8. https://doi.org/10.1038/ nature 13045

23. Vezzoli A, Bonadies N, Allen MD, Freund SM, Santiveri CM, Kvinlaug BT, et al. Molecular basis of histone H3K36me3 recognition by the PWWP domain of Brpfl. Nat Struct Mol Biol. 2010;17(5):617-9. https://doi.org/10.1038/nsmb.1797

24. Pradeepa MM, Sutherland HG, Ule J, Grimes GR, Bickmore WA. Psipl/Ledgf p52 binds methylated histone $\mathrm{H} 3 \mathrm{~K} 36$ and splicing factors and contributes to the regulation of alternative splicing. PLoS Genet. 2012;8(5):e1002717. https://doi.org/10.1371/journal.pgen.1002717

25. Sorenson MR, Jha DK, Ucles SA, Flood DM, Strahl BD, Stevens SW, et al. Histone H3K36 methylation regulates pre-mRNA splicing in Saccharomyces cerevisiae. RNA Biol. 2016;13(4):412-26. https://doi. org/10.1080/15476286.2016.1144009

26. Park IY, Powell RT, Tripathi DN, Dere R, Ho TH, Blasius TL, et al. Dual Chromatin and Cytoskeletal Remodeling by SETD2. Cell. 2016;166(4):950-62. https://doi.org/10.1016/j.cell.2016.07.005

27. Park IY, Chowdhury P, Tripathi DN, Powell RT, Dere R, Terzo EA, et al. Methylated $\alpha$-tubulin antibodies recognize a new microtubule modification on mitotic microtubules. MAbs. 2016;8(8):1590-1597. https://doi.org/10.1080/19420862.2016.1228505

28. Chen K, Liu J, Liu S, Xia M, Zhang X, Han D, et al. Methyltransferase SETD2-Mediated Methylation of STAT1 Is Critical for Interferon Antiviral Activity. Cell. 2017;170(3):492-506.el4. https://doi. org/10.1016/j.cell.2017.06.042

29. Xie P, Tian C, An L, Nie J, Lu K, Xing G, et al. Histone methyltransferase protein SETD2 interacts with p53 and selectively regulates its downstream genes. Cell Signal. 2008;20(9):1671-8. https://doi. org/10.1016/j.cellsig.2008.05.012

30. Swerdlow SH, Campo E, Harris NL, Jaffe ES, Pileri SA, Stein H, et al. World Health Organization Classification of Tumours of Tumours of Haematopoietic and Lymphoid Tissues. Revised 4th ed. International Agency for Research on Cancer; Lyon, France: 2017. 
31. Roberti A, Dobay MP, Bisig B, Vallois D, Boéchat C, Lanitis E, et al. Type II enteropathy-associated T-cell lymphoma features a unique genomic profile with highly recurrent SETD2 alterations. Nat Commun. 2016;7:12602. https://doi.org/10.1038/ncomms12602

32. Tomita S, Kikuti YY, Carreras J, Sakai R, Takata K, Yoshino T, et al. Monomorphic Epitheliotropic Intestinal T-Cell Lymphoma in Asia Frequently Shows SETD2 Alterations. Cancers (Basel). 2020;12(12):3539. https://doi.org/10.3390/cancers12123539

33. Moffitt AB, Ondrejka SL, McKinney M, Rempel RE, Goodlad JR, Teh CH, et al. Enteropathyassociated T cell lymphoma subtypes are characterized by loss of function of SETD2. J Exp Med. 2017;214(5):1371-86. https://doi.org/10.1084/jem.20160894

34. McKinney M, Moffitt AB, Gaulard P, Travert M, De Leval L, Nicolae A, et al. The Genetic Basis of Hepatosplenic T-cell Lymphoma. Cancer Discov. 2017;7(4):369-79. https://doi.org/10.1158/21598290.CD-16-0330

35. Mar BG, Bullinger LB, McLean KM, Grauman PV, Harris MH, Stevenson K, et al. Mutations in epigenetic regulators including SETD2 are gained during relapse in paediatric acute lymphoblastic leukaemia. Nat Commun. 2014;5:3469. https://doi.org/10.1038/ncomms4469

36. Xiao H, Wang LM, Luo Y, Lai X, Li C, Shi J, et al. Mutations in epigenetic regulators are involved in acute lymphoblastic leukemia relapse following allogeneic hematopoietic stem cell transplantation. Oncotarget. 2016;7(3):2696-708. https://doi.org/10.18632/oncotarget.6259

37. Mar BG, Chu SH, Kahn JD, Krivtsov AV, Koche R, Castellano CA, et al. SETD2 alterations impair DNA damage recognition and lead to resistance to chemotherapy in leukemia. Blood. 2017;130(24):2631-41. https://doi.org/10.1182/blood-2017-03-775569

38. Zhu X, He F, Zeng H, Ling S, Chen A, Wang Y, et al. Identification of functional cooperative mutations of SETD2 in human acute leukemia. Nat Genet. 2014;46(3):287-93. https://doi.org/10.1038/ ng.2894

39. Zhang J, Ding L, Holmfeldt L, Wu G, Heatley SL, Payne-Turner D, Easton J, et al. The genetic basis of early T-cell precursor acute lymphoblastic leukaemia. Nature. 2012;481(7380):157-63. https://doi. org/10.1038/nature 10725

40. Li W, Kats A, Cooley LD, Farooqi MS, August K. T-lymphoblastic leukemia/lymphoma with interfollicular growth pattern and Castleman-like morphologic features. Journal of Hematopathology. 2021; 14 (2):163-169. https://doi.org/10.1007/s12308-021-00446-8

41. Parker H, Rose-Zerilli MJ, Larrayoz M, Clifford R, Edelmann J, Blakemore S, et al. Genomic disruption of the histone methyltransferase SETD2 in chronic lymphocytic leukaemia. Leukemia. 2016;30(11):2179-86. https://doi.org/10.1038/leu.2016.134

42. Zhang J, Grubor V, Love CL, Banerjee A, Richards KL, Mieczkowski PA, et al. Genetic heterogeneity of diffuse large B-cell lymphoma. Proc Natl Acad Sci U S A. 2013;110(4):1398-403. https://doi. org/10.1073/pnas.1205299110 